\title{
Cortisol: mediciones de laboratorio y aplicación clínica
}

\author{
Cortisol: Laboratory measurements and clinical application
}

\author{
Sara Hernández-Quiceno $M D^{1}$, Esteban Uribe-Bojanini $M D^{2}$, \\ Juan M. Alfaro-Velásquez MD³, Germán Campuzano-Maya MD4, \\ Lina M. Salazar-Peláez $P h D^{5}$
}

Resumen: la medición del cortisol total en sangre ha sido parte fundamental en el estudio del eje hipotálamo-hipófisis-adrenal y de sus alteraciones, tales como el síndrome de Cushing y la insuficiencia adrenal. El cortisol circula en plasma en su mayoría unido a proteínas, pero su fracción libre es la biológicamente activa y se puede medir en sangre, orina y saliva. La secreción de cortisol no es homogénea durante el día, por el contrario, está regida por un ritmo circadiano, que a su vez, se puede ver afectado por diferentes estresores físicos y psicológicos. Por esta razón, se cuenta con pruebas como el cortisol en orina de 24 horas que permite evaluar la producción diaria de cortisol. También es posible realizar pruebas funcionales como la supresión de cortisol con dexametasona para el estudio del síndrome de Cushing y la prueba de estímulo con la hormona adrenocorticotropa (ACTH) como parte del estudio de la insuficiencia adrenal. Esta revisión tiene como objetivo realizar una puesta al día sobre los diferentes métodos para medir el cortisol como parte del estudio del eje hipotálamo-hipófisis-adrenal, haciendo énfasis en su utilidad para el diagnóstico de condiciones patológicas endocrinas.

\footnotetext{
${ }^{1}$ Médica, Pediatra, Endocrinóloga pediatra, Universidad de Antioquia, Pediatría Universidad CES. Medellín, Colombia. Correspondencia: Calle 19A No. 44-25, Torre Médica Salud y Servicios, consultorio 1810. Teléfono: (57 4) 3223883. Correo electrónico: endocrino@doctorasarahernandez.com

${ }^{2}$ Médico, residente en Dermatología, Universidad CES. Medellín, Colombia.

${ }_{3}^{3}$ Médico, Pediatra, Endocrinólogo pediatra. Director Departamento de Endocrinología Pediátrica, Universidad de Antioquia. Medellín, Colombia.

${ }^{4}$ Médico, especialista en Hematología y Patología Clínica. Docente Ad Honorem, Facultad de Medicina, Universidad de Antioquia. Médico y Director, Laboratorio Clínico Hematológico. Medellín, Colombia

${ }^{5}$ Bacterióloga y laboratorista clínica, MSc en Biotecnología, PhD en Ciencias de la Salud. Docente de la Facultad de Medicina de la Universidad CES. Medellín, Colombia

Conflicto de intereses: los autores declaran que no tienen conflicto de intereses

Medicina \& Laboratorio 2016; 22: 147-164

Módulo 2 (Endocrinología), número 14. Editora Médica Colombiana S.A. $2016^{\circ}$

Recibido el 05 de marzo de 2016; aceptado el 03 de mayo de 2016
} 
Palabras clave: cortisol, orina, suero, saliva, globulina de unión a corticosteroide, síndrome de Cushing, insuficiencia suprarrenal.

Abstract: Total cortisol measurement in blood has been a fundamental part in the study of the hypothalamic-pituitary-adrenal axis, and of its disorders, such as Cushing syndrome and adrenal insufficiency. Cortisol circulates in the plasma mainly bound to proteins, but the free fraction is the biologically active one, which can be measure in blood, urine, and saliva. Cortisol secretion is not homogeneous throughout the day; instead, secretion is governed by a circadian rhythm that also can be affected by different physical and psychological stressors. For this reason, other tests such as 24-hour urinary cortisol are available, which evaluates the daily production of cortisol. Functional tests such as cortisol suppression with dexamethasone for the Cushing's syndrome study and the ACTH stimulation test as part of adrenal insufficiency study can also be performed. This review aims to perform an update on the different cortisol measuring methods as part of the study of hypothalamic - pituitary adrenal axis, emphasizing in their use to endocrine diseases diagnosis.

Key words: Cortisol, urine, serum, saliva, corticosteroid-binding globulin, Cushing syndrome, adrenal insufficiency.

Hernández-Quiceno S, Uribe-Bojanini E, Alfaro-Velásquez JM, Campuzano-Maya G, Salazar-Peláez LM. Cortisol: mediciones de laboratorio y aplicación clínica. Medicina \& Laboratorio 2016; 22: 147-164.

E I eje hipotálamo-hipófisis-adrenal y su producto final, el cortisol, hacen parte fundamental de nuestro sistema adaptativo para el mantenimiento de las funciones vitales durante la exposición a diferentes circunstancias ambientales o estresores. El eje inicia en el hipotálamo donde se produce la hormona liberadora de corticotropina ( $\mathrm{CRH}$; del inglés, Corticotropin Releasing Hormone), la cual, por medio del sistema portahipofisiario, entra en contacto con las células corticotropas de la hipófisis anterior [1].

La hormona antidiurética actúa de forma sinérgica con la hormona liberadora de corticotropina para estimular la producción de la hormona adrenocorticotropa (ACTH; del inglés, Adrenocorticotropic Hormone) por el corticotropo [2] (véase figura 1). Una vez en la sangre, la hormona adrenocorticotropa alcanza la corteza suprarrenal donde estimula la formación de colesterol libre, al mismo tiempo que incrementa la captación de lipoproteínas. Este proceso induce el paso limitante, es decir, el suministro de colesterol a la enzima que divide su cadena lateral, la P450scc o CYP11A1, para la conversión en $\Delta 5$ pregnenolona, que inicia la esteroidogénesis (véase figura 2) [3]. 
La secreción de hormona liberadora de corticotropina por el hipotálamo está regulada por impulsos de centros superiores y se encuentra influenciada por un marcapasos circadiano. Es así como el estrés físico ( $p$. ej. dolor y frío), emocional o fisiológico (p. ej. hipoglicemia o estado de enfermedad) pueden estimular la secreción de hormona liberadora de corticotropina [5].

El ritmo circadiano de la hormona adrenocorticotropa y del cortisol ocurren en paralelo (véase figura 3). Los niveles de cortisol son bajos al final de la tarde y siguen disminuyendo durante las primeras horas de sueño, momento en el cual pueden llegar a ser indetectables. Los niveles más bajos de cortisol se encuentran entre las 22:00 y las 4:00 [6].

Luego, las concentraciones de cortisol empiezan a aumentar gradualmente hasta alcanzar su pico máximo a las 8:00, a partir de donde vuelven a disminuir gradualmente en el transcurso del día. La ingesta de alimentos también induce pequeños picos de secreción de la hormona [7]; no obstante, el $75 \%$ de la producción diaria de cortisol ocurre entre las 4:00 y las 10:00. Por lo tanto, las mediciones basales de cortisol y hormona adrenocorticotropa están sujetas a una gran variabilidad dependiendo del momento del día en el que fueron tomadas.

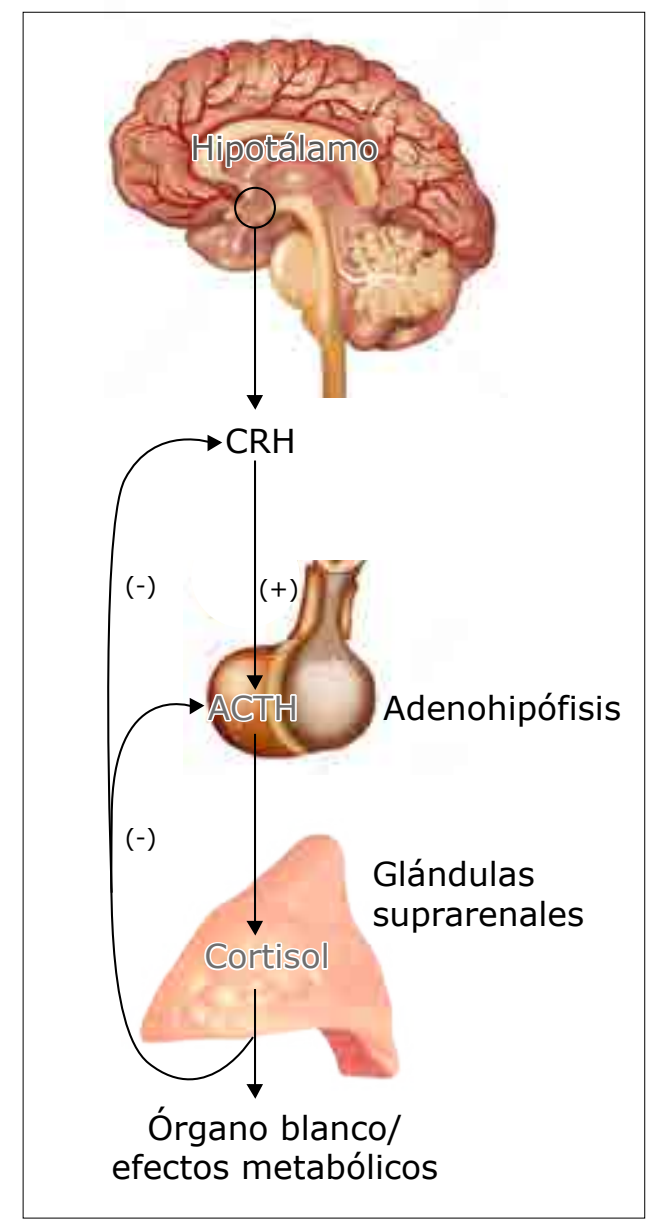

Figura1. Eje hipotálamo-hipófisis-adrenal. La hormona liberadora de corticotropina $(\mathrm{CRH})$, producida en el hipotálamo, estimula la producción de la hormona adrenocorticotropa (ACTH) por la adenohipófisis, la cual actúa en la corteza adrenal estimulando la producción de glucocorticoides. El cortisol, a su vez, tiene un efecto de contrarregulación (regulación negativa) a nivel de la hipófisis y el hipotálamo, disminuyendo la producción de ACTH y $\mathrm{CRH}$, respectivamente. Tomado y modificado de "Glucocorticoides y andrógenos suprarrenales" por Carroll y colaboradores en: Greenspan Endocrinología básica y clínica, por Gardner y colaboradores, 2012, p. 313, México D.F., México: McGraw-Hill. Copyright (c) 2012 por The McGraw-Hill Companies, Inc. [3].

Aunque en general se mantiene un patrón de secreción de cortisol constante, existe una variabilidad intraindividuo e interindividuo y este ritmo se puede ver afectado por situaciones como cambios en el patrón del sueño, la exposición a la luz o a la oscuridad y los cambios en los horarios de alimentación. Además, este ritmo también se puede ver afectado por situaciones menos cotidianas como 


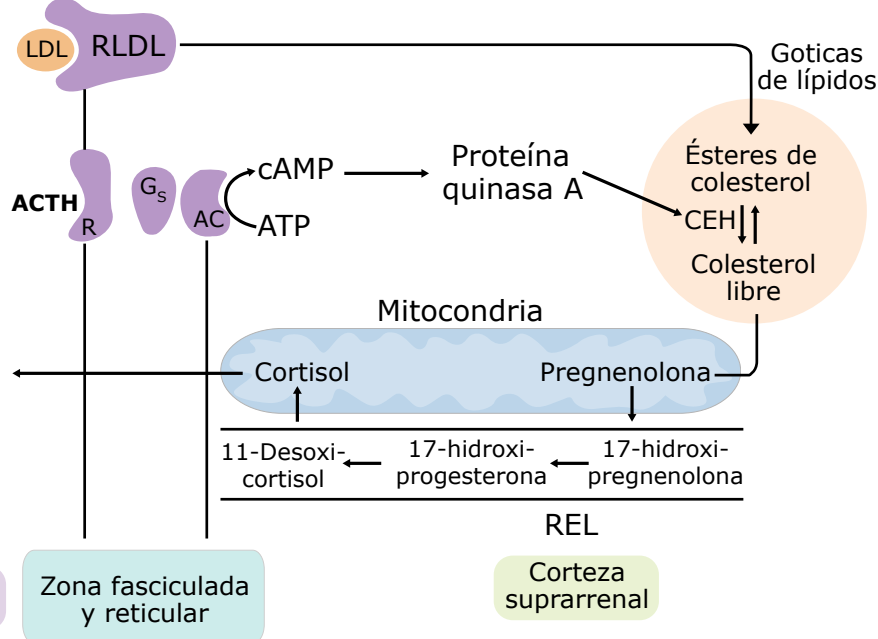

Figura 2. Mecanismo de acción de la hormona adrenocorticotropa (ACTH). La ACTH se une a su receptor $(R)$ en las zonas fasciculada y reticular de la corteza suprarrenal, el cual está acoplado a una proteína G estimuladora (Gs), la cual activa a la enzima adenilato ciclasa (AC) para producir adenosín monofosfato cíclico (CAMP) a partir de ATP y, en consecuencia, aumentar sus niveles. El cAMP activa a la proteína quinasa $A$, cuya función es fosforilar a la enzima colesteril éster hidrolasa (CEH), encargada de la formación de colesterol libre a partir del colesterol tomado del LDL en la circulación y esterificado y almacenado en gotitas de lípidos. Finalmente, el colesterol es transportado a la mitocondria para ser convertido en pregnenolona, lo cual corresponde al paso limitante de la esteroideogénesis. La pregnenolona se mueve hacia el retículo endoplásmico liso (REL), donde es convertida en 17-hidroxi-pregnenolona y luego a 17-hidroxiprogesterona, que es hidroxilada en 11-desoxi-cortisol, que se mueve nuevamente hacia la mitocondria para formar el cortisol que es liberado a la circulación. RLDL= receptor de LDL. Tomado y modificado de "Ganong's Review of Medical Physiology" por Barrett y colaboradores, 2016, p. 359. Copyright (C) 2016 por McGraw-Hill Education [4].

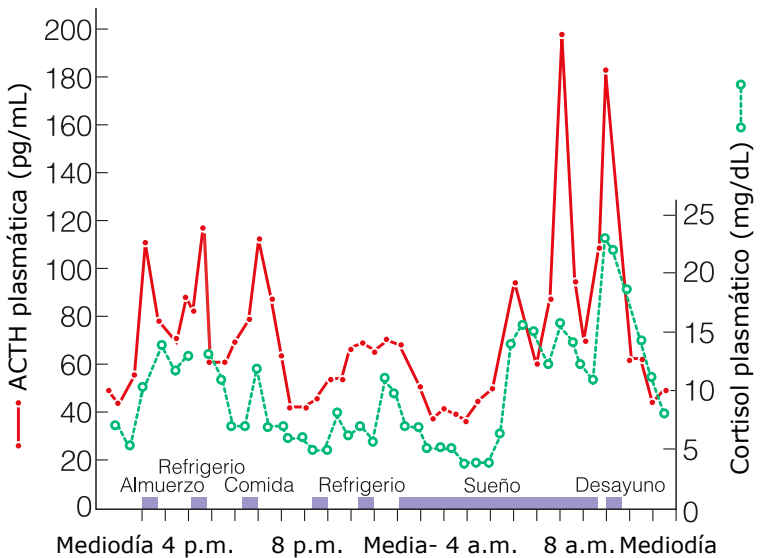

Figura 3. Ritmo circadiano de la hormona adrenocorticotropa (ACTH) y el cortisol. Ambas hormonas presentan un pico en la mañana antes del despertar y posteriormente con las comidas. Tomado y modificado de "Glucocorticoides y andrógenos suprarrenales" por Carroll y colaboradores en: Greenspan Endocrinología básica y clínica, por Gardner y colaboradores, 2012, p. 291, México D.F., México: McGraw-Hill. Copyright (c) 2012 por The McGraw-Hill Companies, Inc. [3]. 
son el estrés físico por trauma o cirugías, el estrés psicológico por ansiedad, depresión o manía, los trastornos del sistema nervioso central y de la hipófisis, la enfermedad hepática, el alcoholismo, la enfermedad renal crónica, el síndrome de Cushing, entre otros [8].

Es por esto que la medición y la interpretación de los valores séricos de cortisol representan un reto tanto para el laboratorio como para el clínico, por lo que resulta relevante profundizar en el fundamento e interpretación de las diferentes pruebas encaminadas a medir la concentración de cortisol en fluidos biológicos, así como sus alcances y limitaciones, para una interpretación adecuada que contribuya a la toma de decisiones clínicas ante un paciente con alteraciones en sus niveles.

\section{Transporte, metabolismo y excreción del cortisol}

Alrededor del $80 \%$ del cortisol es transportado a través del plasma unido a una proteína denominada globulina de unión a corticosteroides (CBG; del inglés Corticosteroid Binding Globulin) o transcortina y, en menor proporción, aproximadamente un $10 \%$, unido a la albúmina. El cortisol unido a proteínas es biológicamente inactivo, por lo que actúa solo como un reservorio. En contraste, la fracción libre o no unida a proteínas, que corresponde al $10 \%$ del cortisol total, es aquella que se une a su receptor en el citoplasma celular para ejercer su función biológica. La concentración plasmática de cortisol libre es aproximadamente de $1 \mu \mathrm{g} / \mathrm{dL}$ y es el regulado por la hormona adrenocorticotropa, mientras que la concentración de cortisol unido a transcortina es de $13 \mu \mathrm{g} / \mathrm{dL}$ [4].

La globulina de unión a corticosteroides es producida en el hígado y se une al cortisol con una afinidad alta. Esta proteína tiene la capacidad de unir hasta 25 $\mu \mathrm{g} / \mathrm{dL}$ de cortisol; no obstante, si la concentración de cortisol se incrementa por encima de este valor, la fracción libre de la hormona aumenta rápidamente hasta exceder su porcentaje habitual del $10 \%$. La hormona progesterona compite con el cortisol por los sitios de unión a la globulina de unión a corticosteroides por lo cual un incremento en los niveles de progesterona, como ocurre en el tercer trimestre del embarazo, puede inhibir la unión del cortisol hasta en un $25 \%$ de los sitios de unión en la globulina de unión a corticosteroides, lo que lleva a la elevación en la fracción del cortisol libre [9].

Así mismo, otros factores pueden incrementar o disminuir la concentración de globulina de unión a corticosteroides. Por ejemplo, el hipertiroidismo, la diabetes o estados que inducen un incremento en la concentración de estrógenos como el embarazo y el uso de anticonceptivos orales aumentan la concentración de la globulina de unión a corticosteroides que, a su vez, disminuye la concentración de la fracción libre de cortisol. Esto, a su vez, estimula la secreción de hormona adrenocorticotropa, lo que induce una realimentación positiva sobre la producción 
de cortisol hasta que este alcanza un nuevo punto de equilibro, donde el cortisol unido y total se encuentra aumentado, mientras que la fracción libre es normal. En cambio, los niveles de globulina de unión a corticosteroides disminuyen en la enfermedad hepática, la enfermedad renal o estados con deficiencia de proteínas. Es así como las pacientes embarazadas tienen un cortisol total elevado, aunque sin síntomas de hipercortisolismo, y los enfermos renales tienen concentraciones bajas de cortisol, pero sin manifestaciones de hipocortisolismo [9].

El cortisol es metabolizado en el hígado y excretado por la orina, siendo menos del $1 \%$ cortisol intacto. El metabolismo del cortisol se puede ver afectado por diversas circunstancias, por ejemplo, se encuentra disminuido en lactantes, ancianos y personas con hipotiroidismo, en las cuales también se reduce su excreción y, por el contrario, se observa un incremento en su metabolismo y excreción en el hipertiroidismo. Otras condiciones que pueden influenciar el metabolismo del cortisol son aquellas relacionadas con la enfermedad hepática o renal, así como el uso de medicamentos que inducen las enzimas hepáticas. No obstante, en términos generales, dichas circunstancias tienen poca repercusión clínica, ya que las concentraciones de cortisol libre suelen permanecer constantes [10].

Por otro lado, debido a que el cortisol posee una similitud molecular con la aldosterona, puede unirse y activar el receptor de mineralocorticoides a nivel renal, por lo que es blanco de la enzima 11- $\beta$-hidroxiesteroide deshidrogenasa, la cual transforma el cortisol en cortisona, un metabolito inactivo. La - $\beta$-hidroxiesteroide deshidrogenasa, en efecto, posibilita la actividad selectiva de los mineralocorticoides; sin embargo, cuando el cortisol sérico se encuentra elevado, como ocurre en la enfermedad de Cushing, este mecanismo es sobrepasado y el cortisol activa los receptores de mineralocorticoides, llevando a hipertensión e hipocalemia [10].

\section{Efectos biológicos del cortisol}

Los receptores para los glucocorticoides se encuentran en casi todos los tejidos del cuerpo, razón por la cual el cortisol presenta efectos sistémicos múltiples: incrementa la gluconeogénesis hepática y favorece la lipólisis del tejido adiposo y la degradación de las proteínas musculares para su utilización como sustratos para la gluconeogénesis; por lo tanto, incrementa los niveles de glucosa en sangre. Además, disminuye la reabsorción del calcio renal y la absorción del calcio, el magnesio y el fósforo en el tracto gastrointestinal. De esta manera, la hipercortisolemia puede inducir defectos en la formación ósea y osteoporosis [11].

En el sistema endocrinológico mismo el cortisol ejerce diversas acciones como disminuir la secreción de la hormona del crecimiento $(\mathrm{GH}$; del inglés, Growth Hormone), reducir la capacidad de respuesta de las células gonadotropas a la hormona liberadora de gonadotropina $(\mathrm{GnRH}$, del inglés, Gonadotropin-Releasing 
Hormone), inhibir la secreción de insulina, aumentar la síntesis de adrenalina y disminuir todas las proteínas de transporte hormonales (p. ej. cortisol, esteroides sexuales y hormonas tiroideas). El cortisol tiene un efecto inmunosupresor y antiinflamatorio, aumenta la contractibilidad cardíaca y la respuesta vascular a los vasoconstrictores. Por último, es fundamental para mantener un equilibrio emocional, aumentar el apetito y suprimir el sueño de movimientos oculares rápidos (REM; del inglés, Rapid Eye Movement) [11].

La medición del cortisol no sólo es útil para el diagnóstico y seguimiento de las diferentes enfermedades del sistema endocrinológico, sino que cada vez se encuentra asociado con mayor frecuencia a diferentes alteraciones del sistema cardiovascular, el sistema nervioso central, las enfermedades infecciosas y las alteraciones en el comportamiento psicosocial. Por ejemplo, la alteración en la respuesta fisiológica del cortisol al despertar (CAR; del inglés, Cortisol Awakening Response) se ha asociado a enfermedades físicas y mentales como el estrés crónico, el autismo y la obesidad, principalmente en el área abdominal $[12,13]$. Un metanálisis realizado por O'Connor y colaboradores (2016) [14] evidenció, además, que en pacientes menores de 40 años los niveles altos de cortisol se asocian a mayores tasas de intentos de suicido. El cortisol se ha propuesto también como un biomarcador en entidades infecciosas como la neumonía, siendo un posible predictor de la mortalidad a corto plazo [15].

\section{Estudios de laboratorio}

Al evaluar el eje hipotálamo-hipófisis-adrenal se cuenta con pruebas estáticas, como la concentración de cortisol basal en sangre, saliva u orina, así como pruebas dinámicas, que nos ofrecen información más precisa, como la medición de cortisol tras el estímulo con hormona adrenocorticotropa o supresión con dexametasona.

\section{Consideraciones básicas previas a los estudios de laboratorio}

Múltiples factores como la edad, el sexo, el estadio puberal, el embarazo, la fase del ciclo menstrual y el ciclo circadiano se han considerado determinantes en la definición de los rangos de referencia y en la interpretación clínica de los niveles de esteroides. El cortisol no se ve fuertemente afectado por el estadio puberal ni por la edad de los pacientes $[16,17]$. Por el contrario, el estrés físico o mental activa el eje por medio de la hormona adrenocorticotropa y lleva al aumento del cortisol. Por este motivo, se deben evitar en la medida de lo posible estresores al momento de la toma de la muestra sanguínea, razón por la cual la medición de cortisol salival, al ser una prueba libre de estrés, se ha popularizado [18].

El tema del ayuno previo a la toma de las muestras sanguíneas es aún controversial; algunos estudios han demostrado elevación del cortisol tras un ayuno pro- 
longado, mientras que otros han evidenciado su aumento luego de una ingesta de comida. Entretanto, si la comida tiene un alto contenido de carbohidratos el cortisol tiende a disminuir [19-21]. Debido a esto, para garantizar la reproducibilidad de las medidas y para excluir los efectos de la ingesta de alimentos se recomienda realizar las tomas sanguíneas para la medición del cortisol en ayuno [22].

\section{Cortisol basal en suero o plasma}

La medición de cortisol en suero o plasma puede ser de gran utilidad en el diagnóstico de la insuficiencia adrenal o el hipercortisolismo, aunque se deben tener en cuenta las variaciones antes mencionadas debidas al ritmo circadiano [23].

\section{Métodos de medición}

Las principales técnicas empleadas por la mayoría de los laboratorios son los inmunoensayos automatizados ( $p$. ej. el ensayo inmunoabsorbente ligado a enzimas o ELISA, el radioinmunoensayo o RIA y la quimioluminiscencia o CLIA) y la cromatografía líquida de alto rendimiento (HPLC) asociada o no a espectrometría de masas (LC/MS); teniendo este último una mayor especificidad en la detección de la molécula de cortisol, pues la separa de sus metabolitos y otros esteroides [24]. Dentro de los inmunoensayos el más ampliamente usado es la quimioluminiscencia.

\section{Valores normales}

En personas sanas, como se mencionó anteriormente, el pico más alto en la concentración de cortisol se observa en las primeras horas de la mañana. En efecto, en los adultos a las 8:00 su concentración se encuentra en un rango entre los 8 $\mu \mathrm{g} / \mathrm{dL}$ y $19 \mu \mathrm{g} / \mathrm{dL}$, mientras que a las $16: 00$ está entre $4 \mu \mathrm{g} / \mathrm{dL}$ y $11 \mu \mathrm{g} / \mathrm{dL}$ (véase tabla 1). Las concentraciones de cortisol llegan a su valor más bajo alrededor de la medianoche y a las 04:00 alcanza valores inferiores a $5 \mu \mathrm{g} / \mathrm{dL}$ [25].

Pese a sus variaciones con el ciclo circadiano, las mediciones en las concentraciones del cortisol alrededor de su pico máximo, en conjunto con una historia clínica adecuada, pueden ser de gran utilidad para el clínico. Por ejemplo, cuando se sospecha de una insuficiencia adrenal, un cortisol sérico a las 8:00 mayor que 10 $\mu \mathrm{g} / \mathrm{dL}$ hace poco probable el diagnóstico, mientras que un valor inferior a $3 \mu \mathrm{g} / \mathrm{dL}$ lo hace bastante factible [26].

En el caso de los pacientes con síndrome de Cushing los valores de cortisol a las 8:00 se pueden encontrar ligeramente elevados o incluso dentro de los límites normales; sin embargo, también se observan valores más o menos constantes en la concentración de cortisol a lo largo día. En efecto, las concentraciones de la hormona determinadas a las 16:00 son similares a las encontradas a las 8:00. Además, el cortisol a la hora después de dormir o cercano a las 00:00 se encuentra por encima de los 7,5 $\mu \mathrm{g} / \mathrm{dL}$ [26]. 


\begin{tabular}{|c|c|c|}
\hline Prueba & Edad & Valor de referencia \\
\hline \multirow[t]{2}{*}{ Cortisol en suero o plasma } & 1-15 años & $\begin{array}{l}8: 00: 3-21 \mu \mathrm{g} / \mathrm{dL} \\
\text { 16:00: No determinado }\end{array}$ \\
\hline & Adultos (> 15 años) & $\begin{array}{l}\text { 8:00: } 8-19 \mu \mathrm{g} / \mathrm{dL} \\
16: 00: 4-11 \mu \mathrm{g} / \mathrm{dL}\end{array}$ \\
\hline Cortisol libre en suero & - & $\begin{array}{l}8: 00: 0,07-0,93 \mu \mathrm{g} / \mathrm{dL} \\
16: 00: 0,04-0,45 \mu \mu \mathrm{g} / \mathrm{dL} \\
23: 00: 0,04-0,35 \mu \mu \mathrm{g} / \mathrm{dL}\end{array}$ \\
\hline Cortisol salival & - & $\begin{array}{l}8: 00: 0,04-0,56 \mu \mathrm{g} / \mathrm{dL} \\
16: 00:<0,15 \mu \mathrm{g} / \mathrm{dL} \\
23: 00:<0,09 \mu \mathrm{g} / \mathrm{dL}\end{array}$ \\
\hline Cortisol libre en orina de 24 horas & $\begin{array}{l}\text { 1-4 años } \\
5-9 \text { años } \\
10-13 \text { años } \\
>14 \text { años }\end{array}$ & $\begin{array}{l}0,9-8,2 \mu \mathrm{g} / 24 \mathrm{~h} \\
1-30 \mu \mathrm{g} / 24 \mathrm{~h} \\
1-45 \mu \mathrm{g} / 24 \mathrm{~h} \\
3-55 \mu \mathrm{g} / 24 \mathrm{~h}\end{array}$ \\
\hline Cortisol en prueba de supresión con dexametasona $1 \mathrm{mg}$ & - & $<1,8-2,0 \mu \mathrm{g} / \mathrm{dL}$ \\
\hline Cortisol en prueba de supresión con dexametasona 2 mg & - & $<1,8-2,0 \mu \mathrm{g} / \mathrm{dL}$ \\
\hline Cortisol en prueba de estímulo con АCTH & - & $>18-20 \mu \mathrm{g} / \mathrm{dL}$ \\
\hline
\end{tabular}

\section{Cortisol libre en plasma o suero}

Aunque la fracción libre del cortisol constituye la forma biológicamente activa de la hormona, su medición por las diferentes técnicas de laboratorio resulta tanto costosa como dispendiosa, por lo que su uso aún no es de amplia difusión en la práctica clínica.

\section{Métodos de medición}

Actualmente, se considera que el mejor método para separar las fracciones de la hormona unida y libre es el de diálisis de equilibrio, seguido por la determinación de la concentración de la fracción libre mediante radioinmunoensayo [27]. Su principal utilidad clínica está siendo estudiada en los pacientes con insuficiencia adrenal asociada a enfermedad crítica, los cuales poseen una unión a proteínas anormal $[28,29]$.

\section{Valores normales}

En la tabla 1 se presentan los valores normales a las 8:00, 16:00 y 23:00 que han sido reportados para el cortisol libre.

\section{Cortisol salival}

Esta prueba funciona bajo el principio de que el cortisol no unido a proteínas difunde libremente a la saliva $y$, por lo tanto, la medición del cortisol salival va a reflejar de manera más exacta los niveles de cortisol libre en sangre [18]. 
La muestra de saliva se obtiene después de enjuagar la boca con agua, pero sin lavarse los dientes y se toma ubicando un tubo de algodón debajo de la lengua durante un minuto, el cual puede ser almacenado a temperatura ambiente. Existen diversos métodos para la toma de muestra de saliva, pero el tubo de algodón es el más utilizado [30].

\section{Métodos de medición}

Se emplean los mismos métodos usados para la medición del cortisol plasmáticos (RIA, ELISA, HPLC y LC/MS) [31].

\section{Valores normales}

La concentración del cortisol salival también presenta variabilidad en el transcurso del día, con concentraciones hasta de $0,56 \mu \mathrm{g} / \mathrm{dL}$ a las $8: 00$ o hasta $0,1 \mu \mathrm{g} / \mathrm{dL}$ a las 23:00 (véase tabla 1) [32] y la interpretación de los resultados es similar a la del cortisol sérico. De esta manera, los niveles elevados de cortisol salival a las 8:00 sugieren hipercortisolismo, mientras que una concentración baja es indicativa de insuficiencia adrenal [33].

Sin embargo, los inmunoensayos usados para la medición de cortisol salival no poseen una especificidad alta, pues pueden arrojar resultados falsos positivos con otros esteroides como la 17-hidroxiprogesterona. Por tal motivo, los pacientes con hiperplasia adrenal congénita secundaria a la deficiencia de 21-hidroxilasa podrían presentar un cortisol falsamente elevado. Por otro lado, para el diagnóstico del síndrome de Cushing se sugiere un resultado de cortisol salival a las 23:00 mayor que $0,4 \mu \mathrm{g} / \mathrm{dL}[34]$.

\section{Cortisol libre en orina de 24 horas}

El cortisol es excretado en la orina en su forma de fracción libre o no unida a proteínas. Por lo tanto, el cortisol libre en orina de 24 horas es una forma útil de medir los niveles de cortisol, al proporcionar un índice de la producción diaria de la hormona y no como una medida única que depende del ritmo circadiano del ACTH-cortisol. Además, esta determinación no se ve afectada por los niveles de la globulina de unión a corticosteroides, pues determina sólo el cortisol libre [35].

No obstante, la exactitud de los resultados va a depender de una adecuada función renal, así como de una minuciosa toma de la muestra. En efecto, la enfermedad renal disminuye la excreción urinaria de cortisol, de manera directamente proporcional a la gravedad de esta. Así mismo, una recolección de orina inapropiada puede alterar los resultados, por lo que se debe tener en cuenta la tasa de excreción de creatinina, que en hombres debe encontrarse entre 20 y $25 \mathrm{mg} / \mathrm{kg}$ de peso y en mujeres entre 15 y $20 \mathrm{mg} / \mathrm{kg}$ de peso [35] 


\section{Métodos de medición}

En condiciones normales menos del $1 \%$ del cortisol secretado se excreta sin cambios en la orina. Por el contrario, en estados de secreción excesiva de cortisol se excede la capacidad de unión de la globulina de unión a corticosteroides $y$, por lo tanto, se incrementa el cortisol libre en el plasma, lo mismo que su excreción urinaria. El cortisol libre en la orina se mide mediante HPLC, RIA y LC/MS [36].

La recolección de la orina empieza a la primera hora de la mañana, después de haber desocupado la vejiga por primera vez. Se recolecta toda gota de orina a partir de este momento y por las próximas 24 horas, día y noche. La recolección termina con la primera orina de la mañana del día siguiente, que debe ser 10 minutos antes o después de la hora en la que se miccionó por primera vez el día anterior [36].

\section{Valores normales}

La excreción de cortisol urinario en condiciones normales es de $10 \mu \mathrm{g} /$ día a $55 \mu \mathrm{g} /$ día, medida por cromatografía líquida con espectrometría de masas o por inmunoensayos. Estos valores pueden aumentar en sujetos que tienen una ingesta de líquidos elevada y presenta variaciones con la edad (véase tabla 1) [37].

Los niveles de cortisol libre en orina tres veces por encima del valor superior de lo normal son indicativos de hipercortisolismo [38], mientras que niveles bajos sugieren insuficiencia adrenal. Sin embargo, estos se deben correlacionar con el cortisol sérico determinado a las 8:00. Algunos pacientes se pueden encontrar en un tipo de zona gris, cuyos valores están por encima del límite normal, pero no superan tres veces el límite superior del valor de referencia. Estos sujetos se pueden clasificar como pseudoCushing o con hipercortisolismo fisiológico, lo cual puede ocurrir en estados de depresión mayor, el síndrome de ovario poliquístico o la obesidad (véase tabla 2) [39].

\begin{tabular}{ll}
\hline $\begin{array}{l}\text { Tabla 2. Causas de hipercortisolismo fisiológico con y sin características clíni- } \\
\text { cas del síndrome de Cushing }\end{array}$ & $\begin{array}{l}\text { Hipercortisolismo fisiológico } \\
\text { sin características clínicas del síndrome de Cushing }\end{array}$ \\
$\begin{array}{l}\text { Hipercortisolismo fisiológico con posibles } \\
\text { características clínicas del síndrome de Cushing }\end{array}$ & $\begin{array}{l}\text { Estrés físico por dolor, cirugía u hospitalización } \\
\text { Obesidad severa, en especial obesidad centrípeta }\end{array}$ \\
$\begin{array}{l}\text { Estrés psicológico severo o } \\
\text { trastorno depresivo mayor }\end{array}$ & Ejercicio de alto rendimiento \\
\hline Síndrome de ovario poliquístico & Amorexia nervosa \\
\hline Embarazo & Aumento de la globulina de unión a corticosteroides \\
\hline Alcoholismo & Resistencia a los glucocorticoides \\
\hline Diabetes mellitus mal controlada & \\
\hline Tomado de "Pseudo-Cushing states" por Romanholi y Salgado, 2007. Creative Commons Attribution License, 2007 [39]. \\
\hline
\end{tabular}




\section{Nuevas técnicas de medición de cortisol}

Como se mencionó previamente, la técnica de cromatografía líquida con espectrometría de masas en tándem tiene una mayor especificidad en la detección de la molécula de cortisol y tiene la ventaja de no tener reacción cruzada con otros esteroides. Además, permite la estandarización para minimizar la variabilidad intra e interlaboratorio y la inexactitud. Por estos motivos se ha propuesto como la técnica estándar de referencia para la medición de los niveles de cortisol en suero, orina y plasma [40-42].

La elevación en los niveles de cortisol a largo plazo desempeña un papel crucial en diferentes condiciones patológicas como el estrés crónico, pero la medición de esos niveles crónicamente elevados en sangre, saliva y orina ha sido limitada. Por este motivo, la medición de sus concentraciones en el pelo se ha propuesto como una opción para valorar los niveles de cortisol sistémico de los últimos meses [43]. Esta técnica aún requiere estudios adicionales para su validación, pero es promisoria [44].

\section{Prueba de supresión con dexametasona}

La prueba de supresión con dexametasona es útil para diferenciar el síndrome de Cushing como causa primaria de hipercortisolismo. La dexametasona se une con alta afinidad a los receptores de glucocorticoides, por lo que inhibe la producción y secreción de la ACTH y, por consiguiente, del cortisol [45].

La dexametasona es el glucocorticoide ideal para estudiar el eje adrenal, ya que es 30 a 40 veces más potente que el cortisol y no afecta la medición de dicha hormona por los inmunoensayos usados actualmente. Los anticuerpos empleados en los inmunoensayos para cortisol están dirigidos contra el anillo $D$ de la molécula y reaccionan muy pobremente con la dexametasona, la cual tiene una modificación en este anillo [46].

En este momento se cuenta con dos protocolos para su realización que se describen a continuación. En ambos casos se debe suspender el uso de anticonceptivos o medicamentos que contengan estrógenos por lo menos seis semanas previas a la prueba [46], lo cual puede ser una gran limitante en mujeres en edad fértil.

- Prueba de $1 \mathrm{mg}$ en la noche: se realiza la administración de $1 \mathrm{mg}$ de dexametasona por vía oral, entre las 23:00 y 00:00. La medición del cortisol sérico se realiza en la mañana del día siguiente a las 8:00. Se considera normal una supresión del cortisol cuando su concentración es menor que 1,8-2,0 $\mu \mathrm{g} / \mathrm{dL}$ (véase tabla 1). Si dicha supresión no se logra es indicativo del síndrome de Cushing, el cual debe ser confirmado con otra prueba [47]. Previamente, se usaba un punto de corte de $5 \mu \mathrm{g} / \mathrm{dL}$, pero el $15 \%$ de los pacientes con síndrome de Cushing suprimían a este nivel, resultando en falsos negativos [46]. 
- Prueba estándar de 2 mg en dos días: se administran 0,5 mg de dexametasona vía oral cada 6 horas hasta completar ocho dosis (dos días). La medición del cortisol sérico se realiza después de 2 o 6 horas de administrada la última dosis. El punto de corte para el diagnóstico de síndrome de Cushing es el mismo que con la prueba de $1 \mathrm{mg}$ de dexametasona (véase tabla 1) [47].

La prueba de $1 \mathrm{mg}$ de dexametasona se usa para evaluar la no supresión del cortisol por incidentalomas adrenales e identificar el paciente con síndrome de Cushing debido a cualquier causa. La prueba de $2 \mathrm{mg}$ de dexametasona, en cambio, es útil para diferenciar los pacientes con enfermedad de Cushing (síndrome de Cushing causado por hipersecreción hipofisiaria de ACTH) de los pacientes con síndrome de ACTH ectópica (síndrome de Cushing causado por secreción de ACHT extrahipofisiaria). Los pacientes con enfermedad de Cushing pueden ser sensibles a la supresión con dexametasona a dosis más altas, a diferencia de lo observado en la secreción ectópica de ACTH [48].

\section{Prueba de estímulo con ACTH}

Como ya se ha mencionado, el cortisol basal tiene grandes limitaciones dado la influencia del ritmo circadiano, así como por sus amplios rangos de normalidad. Dentro del estudio de una insuficiencia adrenal un cortisol a las 8:00 normal no descarta la enfermedad, ya que los pacientes afectados pueden mantener niveles basales de cortisol normales, pero desarrollar una pobre respuesta al estrés.

Un cortisol sérico a las 8:00 por encima de $11 \mu \mathrm{g} / \mathrm{dL}$ o salival por encima de 0,56 $\mu \mathrm{g} / \mathrm{dL}$ hace muy poco probable una insuficiencia adrenal. En cambio, un valor de cortisol sérico a las 8:00 por debajo de los $3 \mu \mathrm{g} / \mathrm{dL}$ o salival inferior a 0,15 $\mu \mathrm{g} / \mathrm{dL}$ apoya fuertemente el diagnóstico. Sin embargo, en la zona gris entre $3 \mu \mathrm{g} / \mathrm{dL}$ y 11 $\mu \mathrm{g} / \mathrm{dL}$, que no descarta la presencia insuficiencia adrenal, la prueba de estímulo con ACTH resulta ser de gran utilidad [49].

La prueba de estímulo con ACTH evalúa la respuesta del cortisol a la administración exógena de ACTH. El agente usado para esta prueba es la consintropina, que corresponde a una ACTH sintética con la misma potencia de la ACTH nativa. Existen dos protocolos con diferentes dosis de $\mathrm{ACTH}$, como se expondrá más adelante. Con ambos se alcanzan niveles suprafisiológicos de ACTH de $60.000 \mathrm{pg} / \mathrm{mL}$ para la dosis alta y de $1.900 \mathrm{pg} / \mathrm{mL}$ para la dosis baja [50]. Siendo los niveles fisiológicos de 50 $\mathrm{pg} / \mathrm{mL}$ como máximo. Esta prueba se prefiere realizar en las horas de la mañana.

- Prueba de estímulo con ACTH a dosis altas: la dosis de ACTH es de $250 \mu \mathrm{g}$ y los niveles de cortisol sérico se miden antes y a los 30 y 60 minutos después de la administración de la ACTH. Además, se puede administrar vía intramuscular 
o intravenosa, ya que con las dosis altas ambas logran niveles farmacológicos de ACTH a los 60 minutos [51].

- Prueba de estímulo con ACTH a dosis bajas: la dosis de ACTH es de $1 \mu \mathrm{g}$. El cortisol sérico se determina antes y 30 minutos después de la administración de ACTH. Teóricamente, esta prueba es más sensible, ya que usa dosis más cercanas al rango fisiológico de la ACTH, captando algunos pacientes con una insuficiencia adrenal leve que responden al estímulo a dosis altas, pero no lo hacen de manera adecuada con la dosis baja [52].

Esta prueba, además, posee una buena reproducibilidad [53]. La ruta de administración de la ACTH debe ser intravenosa, ya que no se ha estudiado si la concentración plasmática de ACTH tras la inyección intramuscular a dosis de $1 \mu \mathrm{g}$ alcanza los niveles farmacológicos adecuados. Según la guías para el diagnóstico y tratamiento de la insuficiencia adrenal primaria, publicadas en el año 2016, la dosis de cosintropina debe ser para adultos y niños mayores de 2 años de $250 \mu \mathrm{g}$, para menores de 2 años $125 \mu \mathrm{g}$ y para menores de 1 año $15 \mu \mathrm{g} / \mathrm{kg}$ de peso [54].

\section{Valores normales}

Una función adrenal normal antes de la administración de la ACTH debe mostrar valores de cortisol sérico mayores que 18-20 $\mu \mathrm{g} / \mathrm{dL}$. Después de la administración de ACTH la mínima respuesta esperada del cortisol sérico es de $18 \mu \mathrm{g} / \mathrm{dL}$ para la prueba con dosis bajas y de $20 \mu \mathrm{g} / \mathrm{dL}$ para la prueba con dosis altas [55]. Los valores por debajo de $16-18 \mu \mathrm{g} / \mathrm{dL}$ son bastante sugestivos de insuficiencia adrenal. También se debe evaluar el delta del cortisol, ya que debe haber un incremento del cortisol tras la administración de la ACTH en al menos 3,3 $\mu \mathrm{g} / \mathrm{dL}$, incluso de $6 \mu \mathrm{g} / \mathrm{dL}$, con respecto al valor basal. Ambas pruebas de estímulo, con dosis altas y bajas de la ACTH, tienen una precisión diagnóstica similar y son bastante útiles para detectar la insuficiencia adrenal secundaria, pero, en caso de ser negativas, no la descartan [56]. Esta prueba no tiene utilidad en el diagnóstico de hipercortisolismo.

\section{Conclusión}

El cortisol es una hormona que desempeña un papel fundamental en el metabolismo y la supervivencia del ser humano. Así mismo, su secreción se puede ver afectada por múltiples situaciones tanto de índole fisiológico como patológico, llevando a estados de déficit o exceso de glucocorticoides (véase tabla 3). En este manuscrito se presentaron los diferentes métodos de medición del cortisol y sus fracciones, exponiendo sus ventajas y limitaciones, y las posibles variaciones en la medición según circunstancias fisiológicas y externas propias de las técnicas de laboratorio que sean de utilidad para la adecuada toma de decisiones clínicas ante un paciente con alteraciones en sus niveles. 


\begin{tabular}{ll}
\hline $\begin{array}{l}\text { Tabla 3. Valores alterados de cortisol sérico en diferentes condiciones } \\
\text { fisiológicas y patológicas }\end{array}$ & Cortisol alto (> $25 \mu \mathrm{g} / \mathrm{dL})$ \\
\hline Cortisol bajo (< $5 \mu \mathrm{g} / \mathrm{dL})$ & Uso de anticonceptivos orales \\
\hline Muestras en horas de la tarde/noche & Embarazo \\
\hline $\begin{array}{l}\text { Tratamiento con glucocorticoides } \\
\text { Panhipopituitarismo }\end{array}$ & Síndrome de Cushing \\
\hline Enfermedad de Addison & Estrés físico por cirugía o trauma \\
\hline Hiperplasia adrenal congénita & Resistencia del receptor de glucocorticoides \\
\hline $\begin{array}{l}\text { Deficiencia aislada de ACTH } \\
\text { Posquirúrgico de síndrome de Cushing }\end{array}$ \\
$\begin{array}{l}\text { Tomado y modificado de "A critical analysis of cortisol measurements: an update" por Maidana y colaboradores, 2013, } \\
\text { p. 582. Copyright (c) 2016 por Medicina Buenos Aires-Combatientes de Malvinas [35]. }\end{array}$ \\
\hline
\end{tabular}

\section{Bibliografía}

1. Herman JP, Cullinan WE. Neurocircuitry of stress: central control of the hypothalamo-pituitary-adrenocortical axis. Trends Neurosci 1997; 20: 78-84.

2. Hauger RL, Aguilera G. Regulation of pituitary corticotropin releasing hormone $(\mathrm{CRH})$ receptors by $\mathrm{CRH}$ : interaction with vasopressin. Endocrinology 1993; 133: 1708-1714.

3. Carroll TB, Aron DC, Findling JW, Tyrrell JB. Glucocorticoides y andrógenos suprarrenales. In: Gardner DG, Shoback D, eds. Greenspan Endocrinología básica y clínica (ed 9a). México D.F., México: McGraw-Hill; 2012: 285-329.

4. Barrett KE, Barman SM, Boitano S, Brooks HL. Ganong's Review of Medical Physiology (ed 25a). China: McGraw-Hill; 2016.

5. Orth DN. Corticotropin-releasing hormone in humans. Endocr Rev 1992; 13: 164-191.

6. Linkowski $\mathbf{P}$, Mendlewicz $\mathbf{J}$, Leclercq $\mathbf{R}$, Brasseur M, Hubain P, Golstein $J$, et al. The 24-hour profile of adrenocorticotropin and cortisol in major depressive illness. J Clin Endocrinol Metab 1985; 61: 429-438.

7. Krieger DT, Allen W, Rizzo F, Krieger HP. Characterization of the normal temporal pattern of plasma corticosteroid levels. J Clin Endocrinol Metab 1971; 32: 266-284.

8. Becker KL. Principles and Practice of Endocrinology and Metabolism (ed 3a). Filadelfia, Estados Unidos: Lippincott Williams \& Wilkins; 2001.

9. Melmed S, Polonsky KS, Larsen PR, Kronenberg HM. Williams Textbook of Endocrinology (ed 12a). Filadelfia, Estados Unidos: Elsevier Saunders; 2011.
10. Arlt W, Stewart PM. Adrenal corticosteroid biosynthesis, metabolism, and action. Endocrinol Metab Clin North Am 2005; 34: 293313, viii.

11. Kacsoh B. Endocrine Physiology (ed 1a). Filadelfia, Estados Unidos: McGraw Hill; 2000.

12. Fries E, Dettenborn L, Kirschbaum C. The cortisol awakening response (CAR): facts and future directions. Int J Psychophysiol 2009; 72: $67-73$

13. Sharpley CF, Bitsika V, Andronicos NM, Agnew LL. Is afternoon cortisol more reliable than waking cortisol in association studies of children with an ASD? Physiol Behav 2016; 155: 218-223.

14. O'Connor DB, Ferguson E, Green JA, O'Carroll RE, O'Connor RC. Cortisol levels and suicidal behavior: A meta-analysis. Psychoneuroendocrinology 2016; 63: 370379.

15. Viasus D, Del Rio-Pertuz G, Simonetti AF, Garcia-Vidal C, Acosta-Reyes J, Garavito A, et al. Biomarkers for predicting short-term mortality in community-acquired pneumonia: A systematic review and meta-analysis. J Infect 2016; 72: 273-282.

16. Ceglarek U, Kortz L, Leichtle A, Fiedler GM, Kratzsch J, Thiery J. Rapid quantification of steroid patterns in human serum by on-line solid phase extraction combined with liquid chromatography-triple quadrupole linear ion trap mass spectrometry. Clin Chim Acta 2009; 401: 114-118.

17. Leblhuber F, Neubauer C, Peichl M, Reisecker $F$, Steinparz $F X$, Windhager $E$, et al. Age and sex differences of dehydroepiandrosterone sulfate (DHEAS) and cortisol 
(CRT) plasma levels in normal controls and Alzheimer's disease (AD). Psychopharmacology (Berl) 1993; 111: 23-26.

18. Raff $\mathbf{H}$. Utility of salivary cortisol measurements in Cushing's syndrome and adrenal insufficiency. J Clin Endocrinol Metab 2009; 94: 3647-3655.

19. Knoll E, Muller FW, Ratge D, Bauersfeld $\mathbf{W}, \mathbf{W i s s e r} \mathbf{H}$. Influence of food intake on concentrations of plasma catecholamines and cortisol. J Clin Chem Clin Biochem 1984; 22: 597-602.

20. Cakir M, Sari R, Tosun O, Karayalcin U. Cortisol levels during an oral glucose tolerance test in lean and obese women. Endocr Res 2005; 31: 213-218.

21. Nakajima ST, Gibson M. The effect of a meal on circulating steady-state progesterone levels. J Clin Endocrinol Metab 1989; 69: 917-919.

22. Ceglarek U, Werner M, Kortz L, Korner A, Kiess W, Thiery J, et al. Preclinical challenges in steroid analysis of human samples. J Steroid Biochem Mol Biol 2010; 121: 505512.

23. Cunningham JM, Buxton OM, Weiss RE. Circadian variation in Cushing's disease and pseudo-Cushing states by analysis of $F$ and ACTH pulsatility. J Endocrinol Invest 2002; 25: 791-799.

24. Taylor RL, Grebe SK, Singh RJ. Quantitative, highly sensitive liquid chromatography-tandem mass spectrometry method for detection of synthetic corticosteroids. Clin Chem 2004; 50: 2345-2352.

25. Weitzman ED, Fukushima D, Nogeire C, Roffwarg $H$, Gallagher TF, Hellman L. Twenty-four hour pattern of the episodic secretion of cortisol in normal subjects. J Clin Endocrinol Metab 1971; 33: 14-22.

26. Papanicolaou DA, Yanovski JA, Cutler GB, Jr., Chrousos GP, Nieman LK. A single midnight serum cortisol measurement distinguishes Cushing's syndrome from pseudo-Cushing states. J Clin Endocrinol Metab 1998; 83: 1163-1167.

27. Vogeser $\mathbf{M}$, Mohnle P, Briegel J. Free serum cortisol: quantification applying equilibrium dialysis or ultrafiltration and an automated immunoassay system. Clin Chem Lab Med 2007; 45: 521-525.

28. Huang $\mathbf{W}$, Kalhorn TF, Baillie $\mathbf{M}$, Shen DD, Thummel KE. Determination of free and total cortisol in plasma and urine by liquid chromatography-tandem mass spectrometry. Ther Drug Monit 2007; 29: 215-224.

29. Ho JT, Al-Musalhi H, Chapman MJ, Quach T, Thomas PD, Bagley CJ, et al. Septic shock and sepsis: a comparison of total and free plasma cortisol levels. J Clin Endocrinol Metab 2006; 91: 105-114.

30. Chen YM, Cintron NM, Whitson PA. Longterm storage of salivary cortisol samples at room temperature. Clin Chem 1992; 38: 304.

31. Baid SK, Sinaii N, Wade M, Rubino D, Nieman LK. Radioimmunoassay and tandem mass spectrometry measurement of bedtime salivary cortisol levels: a comparison of assays to establish hypercortisolism. J Clin Endocrinol Metab 2007; 92: 3102-3107.

32. Castro M, Elias PC, Quidute AR, Halah FP, Moreira AC. Out-patient screening for Cushing's syndrome: the sensitivity of the combination of circadian rhythm and overnight dexamethasone suppression salivary cortisol tests. J Clin Endocrinol Metab 1999; 84: 878882.

33. Carroll $\mathbf{T}$, Raff $\mathbf{H}$, Findling $\mathbf{J W}$. Late-night salivary cortisol measurement in the diagnosis of Cushing's syndrome. Nat Clin Pract Endocrinol Metab 2008; 4: 344-350.

34. Sakihara S, Kageyama K, Oki Y, Doi M, Iwasaki Y, Takayasu S, et al. Evaluation of plasma, salivary, and urinary cortisol levels for diagnosis of Cushing's syndrome. Endocr ] 2010; 57: 331-337.

35. Maidana P, Bruno OD, Mesch V. [A critical analysis of cortisol measurements: an update]. Medicina (B Aires) 2013; 73: 579-584.

36. Chan KC, Lit LC, Law EL, Tai MH, Yung CU, Chan MH, et al. Diminished urinary free cortisol excretion in patients with moderate and severe renal impairment. Clin Chem 2004; 50: 757-759.

37. Mericq MV, Cutler GB, Jr. High fluid intake increases urine free cortisol excretion in normal subjects. J Clin Endocrinol Metab 1998; 83: $682-684$

38. Elamin MB, Murad MH, Mullan R, Erickson $D$, Harris $K$, Nadeem $S$, et al. Accuracy of diagnostic tests for Cushing's syndrome: a systematic review and metaanalyses. J Clin Endocrinol Metab 2008; 93: 1553-1562.

39. Romanholi DJ, Salgado LR. [Pseudo-Cushing states]. Arq Bras Endocrinol Metabol 2007; 51: 1303-1313.

40. Hawley JM, Keevil BG. Endogenous glucocorticoid analysis by liquid chromatography-tandem mass spectrometry in routine clinical laboratories. J Steroid Biochem Mol Biol 2016; 162: 27-40.

41. Koal T, Schmiederer D, Pham-Tuan H, Rohring C, Rauh M. Standardized LC-MS/MS based steroid hormone profile-analysis. J Steroid Biochem Mol Biol 2012; 129: 129-138. 
42. Mezzullo M, Fanelli F, Fazzini A, Gambineri A, Vicennati V, Di Dalmazi G, et al. Validation of an LC-MS/MS salivary assay for glucocorticoid status assessment: Evaluation of the diurnal fluctuation of cortisol and cortisone and of their association within and between serum and saliva. J Steroid Biochem Mol Biol 2016; 163: 103-112.

43. Stalder T, Kirschbaum C. Analysis of cortisol in hair--state of the art and future directions. Brain Behav Immun 2012; 26: 10191029.

44. Herane Vives $A$, De Angel $\mathbf{V}$, Papadopoulos A, Strawbridge $R$, Wise $T$, Young AH, et al. The relationship between cortisol, stress and psychiatric illness: New insights using hair analysis. J Psychiatr Res 2015; 70: 38-49.

45. Liddle GW. Tests of pituitary-adrenal suppressibility in the diagnosis of Cushing's syndrome. J Clin Endocrinol Metab 1960; 20: 1539-1560.

46. Findling JW, Raff H, Aron DC. The low-dose dexamethasone suppression test: a reevaluation in patients with Cushing's syndrome. J Clin Endocrinol Metab 2004; 89: 1222-1226.

47. Nieman LK, Biller BM, Findling JW, Newell-Price J, Savage MO, Stewart PM, et al. The diagnosis of Cushing's syndrome: an Endocrine Society Clinical Practice Guideline. J Clin Endocrinol Metab 2008; 93: 1526-1540.

48. Isidori AM, Kaltsas GA, Mohammed S, Morris DG, Jenkins P, Chew SL, et al. Discriminatory value of the low-dose dexamethasone suppression test in establishing the diagnosis and differential diagnosis of Cushing's syndrome. J Clin Endocrinol Metab 2003; 88: 5299-5306.
49. Hagg E, Asplund K, Lithner F. Value of basal plasma cortisol assays in the assessment of pituitary-adrenal insufficiency. Clin Endocrinol (Oxf) 1987; 26: 221-226.

50. Tordjman $\mathbf{K}$, Jaffe A, Greenman $\mathbf{Y}$, Stern $\mathbf{N}$. Comments on the comparison of low and high dose corticotropin stimulation tests in patients with pituitary disease. J Clin Endocrinol Metab 1998; 83: 4530; author reply 4532-4533.

51. Longui CA, Vottero A, Harris AG, Chrousos GP. Plasma cortisol responses after intramuscular corticotropin 1-24 in healthy men. Metabolism 1998; 47: 1419-1422.

52. Dickstein G, Shechner C, Nicholson WE, Rosner I, Shen-Orr Z, Adawi F, et al. Adrenocorticotropin stimulation test: effects of basal cortisol level, time of day, and suggested new sensitive low dose test. J Clin Endocrinol Metab 1991; 72: 773-778.

53. Park YJ, Park KS, Kim JH, Shin CS, Kim SY, Lee HK. Reproducibility of the cortisol response to stimulation with the low dose (1 microg) of ACTH. Clin Endocrinol (Oxf) 1999; 51: 153-158.

54. Bornstein SR, Allolio B, Arlt W, Barthel A, Don-Wauchope A, Hammer GD, et al. Diagnosis and Treatment of Primary Adrenal Insufficiency: An Endocrine Society Clinical Practice Guideline. J Clin Endocrinol Metab 2016; 101: 364-389.

55. Dorin RI, Qualls CR, Crapo LM. Diagnosis of adrenal insufficiency. Ann Intern Med 2003; 139: 194-204.

56. Ospina NS, Al Nofal A, Bancos I, Javed A, Benkhadra K, Kapoor E, et al. ACTH Stimulation Tests for the Diagnosis of Adrenal Insufficiency: Systematic Review and Meta-Analysis. J Clin Endocrinol Metab 2016; 101: 427-434. 


\section{Creemos en los sueños de todos los colombianos que llevan en la sangre confianza}

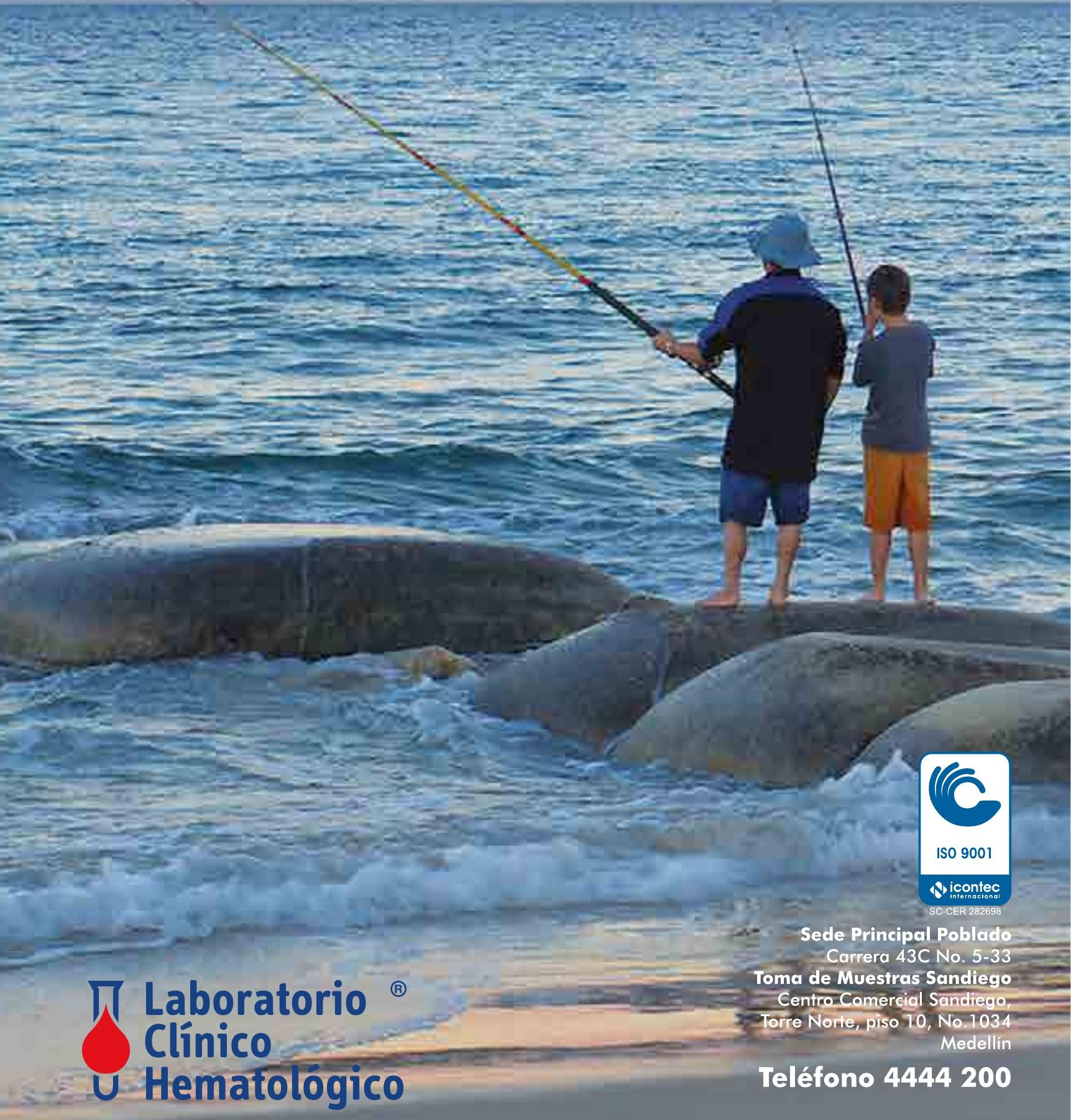

